\title{
NOUVELle
}

\section{Quand les mutants de p53 se livrent à des trafics!}

Claude Caron de Fromentel, Véronique Maguer-Satta
Inserm UMR590, 28 rue Laennec, F-69008, Lyon, France. Université Lyon-1, F-69622, Villeurbanne, France. Centre Léon Bérard, 28 rue Laennec F-69008 Lyon, France. carondef@lyon.fnclcc.fr
> Dans la plupart des cancers solides, le développement de métastases est la principale cause de décès des patients. De ce fait, les mécanismes responsables de la migration des cellules tumorales, un phénomène nécessaire à la fois à l'invasion des tissus adjacents à la tumeur et à la formation des métastases, sont l'objet de nombreuses études, avec pour but le développement de thérapies ciblant les cellules métastatiques. Identifier de nouvelles voies de signalisation permettrait de développer ce type de thérapie.

\section{Mutations de p53 et avantage} prolifératif des cellules tumorales La protéine p53 est codée par le gène TP53, certainement le plus célèbre des gènes suppresseurs de tumeur (voir les deux numéros de Nature Reviews Cancer consacrés aux 30 ans de la découverte de p53 [1, 2]). p53 est un facteur de transcription dont la fonction principale est la protection de l'intégrité du génome. Tout type de stress susceptible de provoquer des altérations génétiques induit l'activation de p53 qui, à son tour, module des voies de signalisation impliquées dans la réparation des lésions ou la mort de la cellule [1]. La fonction de p53 est de ce fait une barrière puissante à l'émergence de cellules présentant des altérations génétiques et donc potentiellement tumorigènes. En conséquence, il n'est pas étonnant que les remaniements du gène TP53 soient l'événement le plus fréquent dans les tumeurs humaines ${ }^{l}$.

TP53 n'est pas le seul gène suppresseur de tumeur remanié dans les tumeurs. Néanmoins, des gènes tels que RBI (retinoblastoma), APC (adenomatous polyposis coli) ou BRCAI (breast cancer l) sont généralement purement et simplement délétés en tout ou en partie (Figure 1). À l'inverse, plus de $70 \%$ des remaniements de TP53 détectés dans les tumeurs sont des mutations faux sens, qui permettent la production d'une protéine ne différant de la protéine sauvage que par un acide aminél [3].

Mais pourquoi maintenir une version mutée d'un gène suppresseur de tumeur

\footnotetext{
${ }^{1}$ http://www-p53.iarc.fr
}

dans une cellule tumorale? La réponse réside dans les propriétés spécifiques des mutants de p53, qui confèrent aux cellules tumorales une prolifération accrue ainsi qu'une meilleure survie, en inhibant la mort programmée par apoptose ou en augmentant la résistance aux agents chimiothérapeutiques. La plupart de ces propriétés sont dues à la capacité des mutants à transactiver, directement ou indirectement, des gènes impliqués dans ces processus, gènes qui ne sont pas transactivés par la protéine sauvage [2].

Néanmoins, un autre mécanisme a été décrit il y a une dizaine d'années, la capacité de la p53 mutée à interagir avec TAp63 et TAp73 et à inhiber leurs fonctions [2]. TAp63 et TAp73 sont des isoformes issues de TP63 et TP73, les deux autres membres de la famille TP53. Elles sont, elles aussi, des facteurs de transcription et des produits de gènes suppresseurs de tumeur. Elles peuvent se substituer à $p 53$ pour induire, par exemple, la mort par apoptose en réponse à un traitement chimiothérapeutique [4, 5]. Dans les cancers de la tête et du

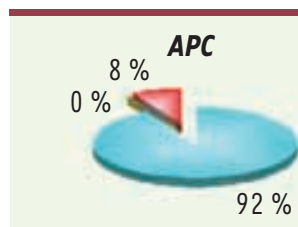

RBI

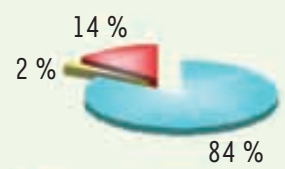

Mutations faux sens

Délétions, insertions. Conservation du cadre de lecture

Délétions, insertions, mutations non sens. Perte du cadre de lecture
Figure 1. Altérations génétiques des gènes suppresseurs de tumeur APC, BRCA1, RB1 et TP53. La plupart des altérations touchant les gènes APC (adenomatosis polyposis coli), RBl (retinoblastoma) et BRCAl (breast cancer l) ne permettent pas la production de la protéine correspondante. $\varepsilon$ n revanche, les altérations touchant le gène TP53 aboutissent dans la plupart des cas à une protéine différant de la protéine sauvage par un seul acide aminé. Données issues de la base de données COSMIC (http://www.sanger.ac.uk/genetics/CGP/cosmic/). 


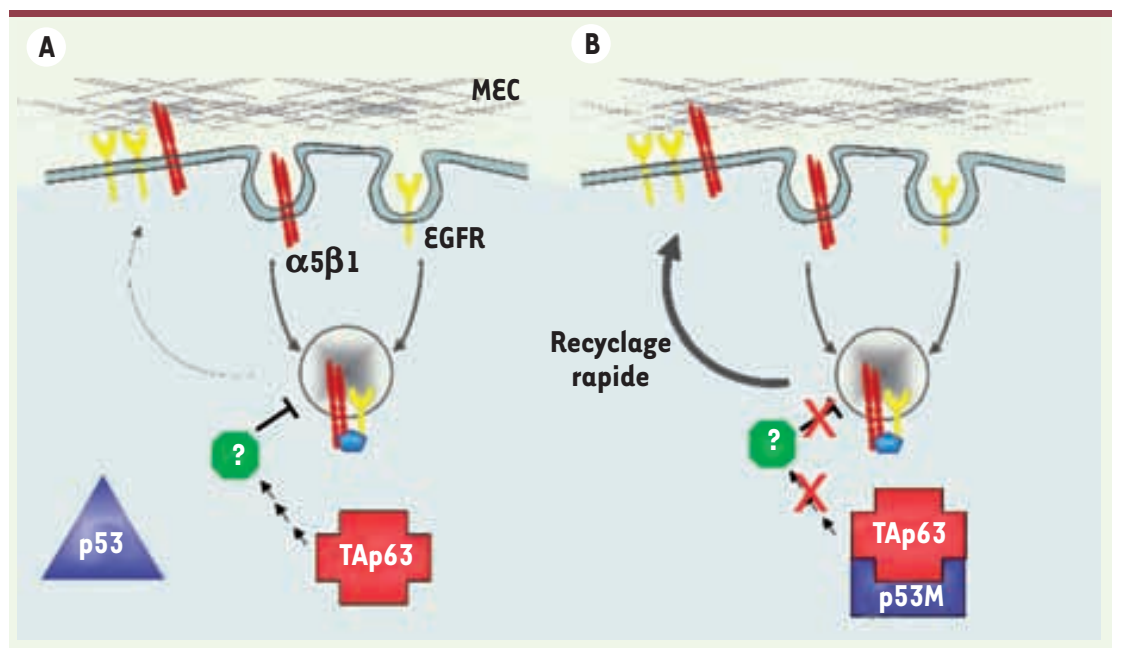

cou, il a été montré que la capacité de certains mutants de p53 à protéger les cellules tumorales contre des agents chimiothérapeutiques est corrélée avec leur capacité à interagir avec TAp73 et à inhiber son activité [6]. Autre exemple, en présence d'un mutant de $p 53$, les protéines $p 63$ et $p 73$ induisent l'expression de l'intégrine $\beta 4$, ce qui a pour effet d'augmenter le pouvoir invasif des cellules tumorales [7].

\section{Mutants de $\mathrm{p} 53$}

et pouvoir métastatique :

le rôle-clé de TAp63

et du recyclage des intégrines

Une nouvelle propriété des mutants de p53, très importante en termes de progression tumorale, a été décrite en 2009, qui cible cette fois-ci la protéine p63. Deux articles ont décrit que certains mutants de p53 favorisent l'invasion et l'apparition de métastases d'une part, en séquestrant une isoforme tronquée de p63, appelée $\Delta \mathrm{Np} 63$ [8] et d'autre part, en inhibant le contrôle exercé par TAp63 sur le trafic intracellulaire des intégrines et d'EGFR, le récepteur de l'EGF (epidermal growth factor) [9].

Les intégrines sont une famille de molécules qui jouent un rôle-clé dans les liaisons entre la cellule et la matrice extracellulaire (MEC) et dans l'activation des voies de signalisation associées. Présentes sous forme d'hétérodimères entre les chaînes de type $\alpha$ et $\beta$ à la surface des cellules, elles sont un lien mécanique entre le cytosquelette d'actine et la $M E C$, permettant à la cellule d'exercer des forces de traction [14]. De plus, elles contrôlent la polymérisation des filaments d'actine et la contractilité cellulaire. Elles sont recyclées par internalisation dans des endosomes [10], puis redirigées vers la membrane plasmique (Figure 2A). Le trafic intracellulaire des intégrines via les endosomes contrôle de nombreux processus liés à l'adhésion, tels que la motilité cellulaire, la cytokinèse et les interactions entre les intégrines et les récepteurs des facteurs de croissance [11].

De façon intéressante, les interactions des intégrines $\beta 1$ avec leurs ligands (MEC) ont été impliquées dans le maintien des cellules souches dans leur niche et dans la régulation de leur quiescence $[15,16]$. Dans ce contexte, la stabilisation de l'expression des intégrines par l'interaction TAp63/p53 mutant pourrait contribuer au maintien et à la survie des cellules souches tumorales impliquées dans les rechutes et les résistances. Dans des cellules plus différenciées, il a été montré que le recyclage des dimères $\alpha 5 \beta$ l contribue à la migration et au pouvoir invasif des cellules tumorales [12].

Muller et al. ont mis en évidence pour la première fois que TAp63 inhibe le recyclage des intégrines et d'EGFR, mais
Figure 2. Recyclage des intégrines $\alpha 5 \beta 1$ et de l'EGFR. Les dimères d'intégrines $\alpha 5 \beta 1$ (jaune) et EGFR (rouge) sont internalisés dans des endosomes en présence de la protéine RCP (rab-coupled protein). Puis, ils sont recyclés ensemble au niveau de la membrane. $A$. En présence d'une protéine $p 53$ sauvage, TAp63 est active et limite le recyclage rapide et couplé des intégrines $\alpha 5 \beta 1$ et d'EGFR. B. En présence d'un mutant de $p 53$, TAp63 est retenue dans un complexe avec $p 53$. Le recyclage d' $\alpha 5 \beta 1$ et d'EGFR est accéléré, ce qui a pour effet d'augmenter la migration, l'invasion et la formation de métastases.

pas leur internalisation. L'expression d'un mutant de p53 bloque l'activité de TAp63, ce qui favorise le recyclage des intégrines et d'દGFR et augmente les capacités de migration et d'invasion des cellules in vitro (Figure 2B). Ceci se traduit in vivo par une invasion accrue et l'apparition de métastases, à partir de xénogreffes de tumeurs humaines qui ne métastasent pas en l'absence de mutant de 553 [9].

\section{Des perspectives}

\section{thérapeutiques réelles}

Bien que de nombreux points restent encore à éclaircir, notamment l'identification des protéines partenaires et gènes cibles de TAp63 impliqués dans ces phénomènes, des approches thérapeutiques peuvent d'ores et déjà être envisagées. D’une part, bloquer les intégrines $\alpha 5 \beta 1$ et/ou l'EGFR pourrait être utilisé pour diminuer le pouvoir invasif de cellules exprimant une p53 mutée. D'autre part, l'utilisation de molécules capables de bloquer l'interaction entre le mutant de p53 et TAp63 pourrait, outre son action inhibitrice sur l'invasion, permettre d'éliminer totalement la tumeur, en restaurant l'activité proapoptotique de TAp63 en réponse à des traitements chimiothérapeutiques. L'efficacité de ce type de molécules a déjà été prouvée dans le cas de p73. En effet, de petits peptides capables de rompre l'association entre un mutant de p53 et 
p73 ont été identifiés, qui permettent à TAp73 de retrouver son activité et d'augmenter ainsi la sensibilité des cellules à la chimiothérapie [13]. L'identification de molécules ayant le même effet sur les complexes entre les mutants de p53 et TAp63 est donc envisageable dans un futur proche. Elles permettraient de cibler les carcinomes issus des épithéliums stratifiés et glandulaires, qui expriment $p 63$ et dont certains figurent parmi les types de tumeurs présentant le pourcentage le plus important de mutations du gène TP5 $3^{2}$. $\diamond$

When p53 mutants

engage in trafficking!

\section{CONFLIT D'INTÉRÊTS}

Les auteurs déclarent n'avoir aucun conflit d'intérêts concernant les données publiées dans cet article.

\footnotetext{
${ }^{2}$ Voir note 1.
}

\section{RÉFÉRENCES}

1. Levine AJ, Oren M. The first 30 years of $p 53$ : growing ever more complex. Nat Rev Cancer 2009; $9: 749-58$.

2. Brosh $R$, Rotter $V$. When mutants gain new powers: news from the mutant $\mathrm{p} 53$ field. Nat Rev Cancer 2009; 9: 701-13

3. Petitjean A, Achatz MI, Borresen-Dale AL, et al. TP53 mutations in human cancers: functional selection and impact on cancer prognosis and outcomes. Oncogene $2007 ; 26: 2157-65$.

4. Douc-Rasy S, Goldschneider D, Million K, Benard J. Interactivité entre $\mathrm{p} 73$ et $\mathrm{p} 53$ dans les cancers : un modèle, le neuroblastome. Med Sci (Paris) 2004 ; 20 : 317-24.

5. Petitjean A, Hainaut P, Caron de Fromentel C. TP63 gene in stress response and carcinogenesis: a broader role than expected. Bull Cancer 2006; 93 : E126-35.

6. Bergamaschi D, Gasco M, Hiller L, et al. p53 polymorphism influences response in cancer chemotherapy via modulation of $\mathrm{p} 73$-dependent apoptosis. Cancer Cell $2003 ; 3$ : 387-402.

7. Bon G, Di Carlo SE, Folgiero V, et al. Negative regulation of beta4 integrin transcription by homeodomain-interacting protein kinase 2 and p53 impairs tumor progression. Cancer Res 2009; $69: 5978-86$

8. Adorno M, Cordenonsi M, Montagner M, et al. A Mutant-p53/Smad complex opposes p63 to empower TGFbeta-induced metastasis. Cell 2009 ; 137 : 87-98.
9. Muller PA, Caswell PT, Doyle B, et al. Mutant p53 drives invasion by promoting integrin recycling. Cell 2009; 139 : 1327-41.

10. Coumailleau F, Babinet C, Cohen-Tannoudji M. Rififyline et recyclage : quel trafic! Med Sci (Paris) 2005; $21: 235-7$.

11. Caswell PT, Vadrevu S, Norman JC. Integrins: masters and slaves of endocytic transport. Nat Rev Mol Cell Biol 2009; 10 : 843-53.

12. Caswell PT, Chan M, Lindsay AJ, et al. Rab-coupling protein coordinates recycling of alpha5betal integrin and EGFRl to promote cell migration in 3D microenvironments. J Cell Biol 2008 ; 183 : 143-55.

13. Di AS, Cortese G, Monti 0 , et al. The disruption of the protein complex mutant $p 53 / p 73$ increases selectively the response of tumor cells to anticancer drugs. Cell Cycle 2008 ; 7 : 3440-7.

14. Albiges-Rizo C, Bouvard D, Bouin AP, et al. La taline : une allure d'haltérophile et la pratique du stretching pour mieux transmettre les forces. Med Sci (Paris) $2009 ; 25: 909-11$

15. Lataillade JJ, Brunet de la Grange P, Le BousseKerdiles C, Uzan G. Les cellules souches ont-elles l'age de leur niche? À la recherche d'un sérum de jouvence... Med Sci (Paris) $2010 ; 26: 582-5$.

16. Bachelard-Cascales $\varepsilon$, Chapellier M, Delay $\varepsilon$, Pochon G, Voeltzel T, Puisieux A, Caron de Fromentel C, Maguer-Satta V. The CDI0-enzyme is a key player to identify and regulate human mammary stem cells. Stem Cells $2010 ; 28$ : 1081-8.

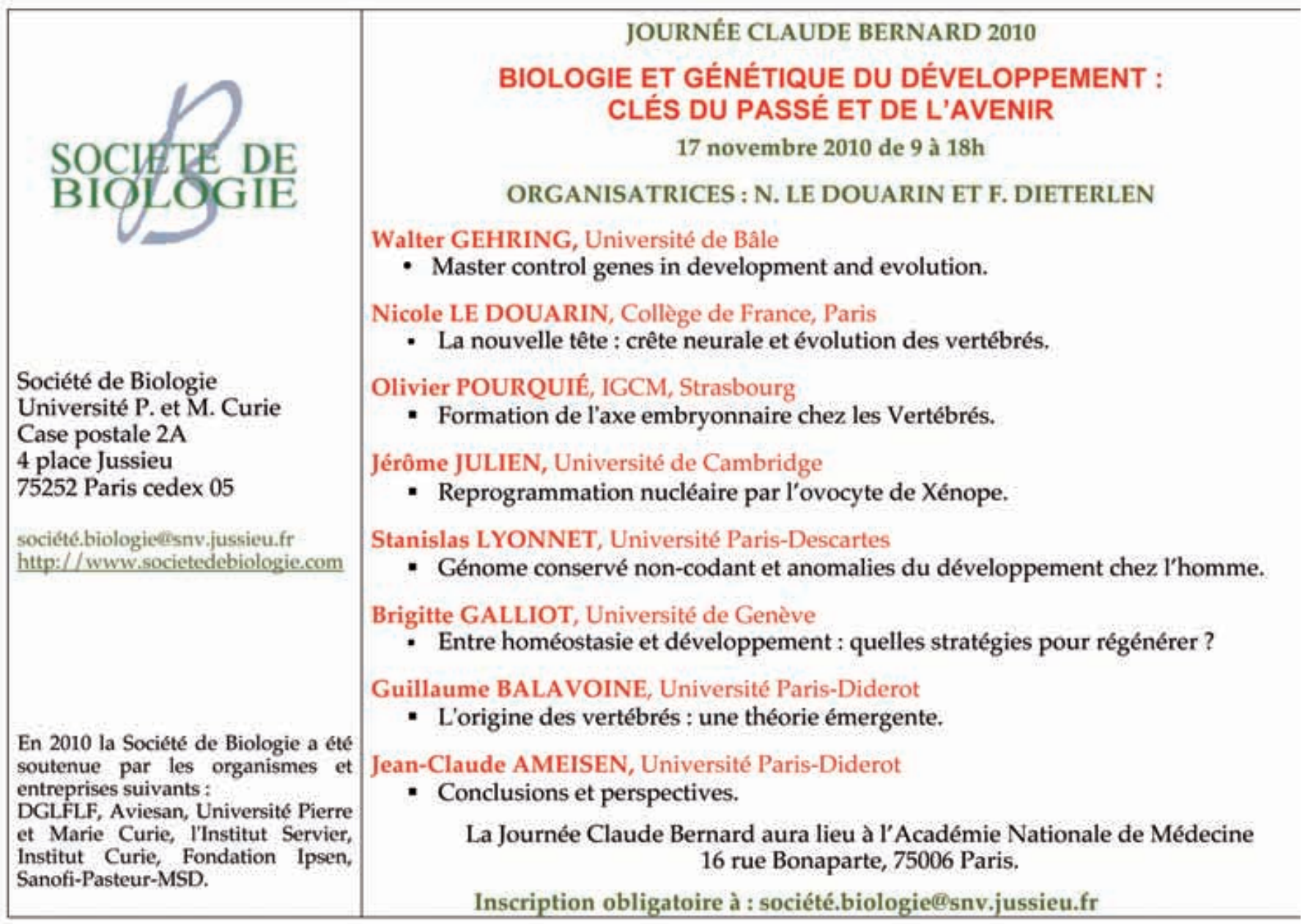

